\title{
Simulation and visualisation of supersonic underexpanded jet interaction with a blunt body and periodic energy input
}

\author{
Ya.V. Khankhasaeva1,A, A.L. Afendikov²,A, A.E. Lutsky3,A, I.S. Menshov4,A, \\ I.A. Znamenskaya5,B \\ A Keldysh Institute of Applied Mathematics \\ (Russian Academy of Sciences), Moscow, Russia \\ B Lomonosov Moscow State University, Russia, Moscow \\ 1 ORCID: oooo-0oo3-2748-2591, hanhyana@mail.ru \\ 2 ORCID: 0000-0002-7880-555X, andre@keldysh.ru \\ 3 ORCID: 0000-0002-4442-0571, lutsky@kiam.ru \\ 4 ORCID: 0000-0003-2300-4007, menshov@kiam.ru \\ 5 ORCID: oooo-0o01-6362-9496, znamen@phys.msu.ru
}

\begin{abstract}
The unsteady flow structure evolution during an interaction of supersonic under expanded jet, a blunt body and periodic energy input has been investigated using the free boundary method on multilevel Cartesian grids with local adaptation based on the wavelet analysis. The grid is restructured according to the various occurring discontinuities.

Periodic energy input first leads to an increase in body drag (due to energy source shockwave reaching the body) then to decrease to a level lower than if no energy input was present. A large amount of discontinuities and their interaction with each other can be observed. Due to the flow around the body being non-uniform (presence of under expanded jet and low- pressure chamber), shock waves occurring from energy input become curved. Shock waves from previous energy inputs interact with those from the next ones and contact discontinuities, which results in structures similar to the Richtmyer-Meshkov instability.

The flow dynamics are illustrated with a series of images and animations which show the distribution of density and pressure, stream lines and mesh structure.

Keywords: computational fluid dynamics, free boundary method, Cartesian grids, mesh refinement, supersonic under expanded jet, periodic energy input.
\end{abstract}

\section{Introduction}

One of the methods for improving the aerodynamic characteristics of prospective aircraft is a controlled influence on the oncoming flow. There are a lot of ways that can be accomplished, in particular by using energy input localized in a small region. The possibility of remote energy input into a supersonic flow is confirmed in many experiments [1-4]. The hightemperature wake is formed behind the energy source with reduced values of the Mach number, total pressure and velocity, which enables the flow regime altering. If the energy source and body are of comparable sizes then the flow around the body is quasi-uniform and drag can be reduced by changing straightforwardly the parameters of the oncoming flow. This oncoming flow requires large energy expense and is impractical. However, energy input even in a relatively small space region can lead to restructuring the bow shock wave ahead of the body. This method of drag reduction is rather efficient.

At this point numerous theoretical and experimental research studies (for instance [1-7]) on the reduction of wave resistance have been performed. It was shown that energy deposition into the flow in front of an aircraft's nose allows reducing the wave resistance by at least several orders of magnitude due to the formation of a cone- shaped separated flow zone in 
front of the nose. The power consumption at the energy input is significantly less than saving engine power which is provided by reducing the resistance. Most of the work is devoted to effects and flow structure.

The majority of the works are concerned with the effects and flow structure occurring during the steady energy input into a uniform supersonic flow. Work [4] though is investigating the effects of impulse energy input into a supersonic under expanded jet flowing past a blunt body. The resulting flow is characterized by spherical plasmoid formation, the discontinuities generated by it, and their action on the bow shock wave in front of the model. The present work's goal is to numerically investigate a similar problem of the interaction of supersonic under expanded jet, a blunt body and energy input, only the energy input is periodic in nature.

Since the resulting flow is unsteady due to periodic energy input and has a lot of discontinuities and their interaction, it makes sense to adapt the grid to the moving features. For this purpose, multilevel Cartesian grids with local adaptation based on wavelet analysis are used [8-10]. For local evaluation of the smoothness of the grid function, a criteria based on wavelet decomposition on local templates is applied, which allows us to clearly see the location of the discontinuities and large gradients, which in turn helps in the understanding of flow physics.

The free boundary method [11,12] is used in the present work to simulate the problem conditions. A Cartesian grid covers both the region of the flow and the area occupied by the solid bodies. To fulfill the boundary condition on the body surface, compensating fluxes of mass, momentum and energy are introduced. One of the major advantages of this approach is the simplicity of grid construction which does not depend on the complexity of the body geometry.

For a deeper understanding of flow physics, visualization tools such as the animation of density and pressure fields with stream traces and shadowgraphs are used to illustrate the flow evolution. They allow us to track the interaction of discontinuities and instabilities.

\section{Problem statement}

In the experiment [4] a supersonic Laval nozzle and a plasma generator operating on the principle of a magnetoplasma compressor (MPC) were placed inside the low- pressure chamber 1 (Fig. 1). The high pressure at the nozzle inlet was created using a compressor. The pressure at the nozzle inlet was supplied by means of a controlled valve through the nozzle 3 . Power was supplied to the magnetoplasma compressor through a pressurized connector 4 . The MPC storage capacitor 5 was charged by a power source with a maximum voltage of $5 \mathrm{kV}$. The energy discharge parameters in the experiment were amperage $I_{\max }=12 k A$, voltage $U=$ $700 \mathrm{~V}$ with discharge current oscillogram displayed on Fig. 1.

The simulation area consists of a low pressure chamber (area 1), a supersonic jet (area 2), nozzle wall and body (Fig. 2). Simulation area constraints are $x \in[-12 ; 4], y \in[0 ; 10]$. Starting parameters are:

1) low pressure chamber, $\rho=1, u=v=0, p=1$

2) supersonic jet with $M=2.4, \rho=2.684424, u=2.444169, v=0, p=2$

The body is a cylinder with a diameter $D=1$ and length $L=4$ with truncated cone head (cone semi- angle $22.69^{\circ}$, truncated diameter $\left.d=0.5625\right)$. The body and the nozzle wall are represented by the free boundary method $[11,12]$. The problem statement and conditions are close to the ones from [4]. The grid has 3 levels, the main grid has a size of $320 x 200$ cells.

The 1st level cells have $\Delta x=\Delta y=0.05$, the next level the cell size is two times smaller than the previous one (the second level $-\Delta x=\Delta y=0.025$, the 3 rd level $-\Delta x=\Delta y=0.0125$ ) (Fig. 2). As in [9], the model of the Euler equations describing the motion of an ideal compressible fluid is used which is solved by the finite volume method. In this work, we are more interested in the discontinuities' interaction, and at high Re number of experiment [4] and the energy discharge time, the viscosity effect can be neglected. The algorithm provides a 
second order approximation on smooth flow domains. The problem was solved in an axisymmetric formulation. The numerical algorithm is described in detail in [10] and used undergoing extensive testing, see [8-10]. The problem was solved in the axially symmetric formulation.
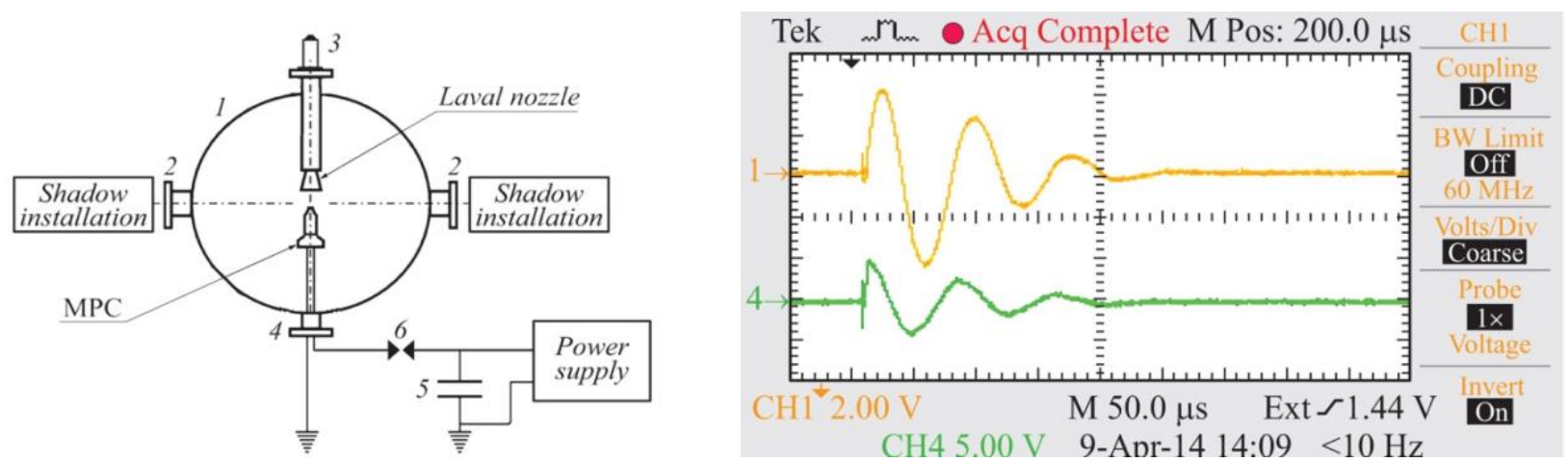

Fig. 1. Experimental setup and discharge current oscillogram taken from [4].
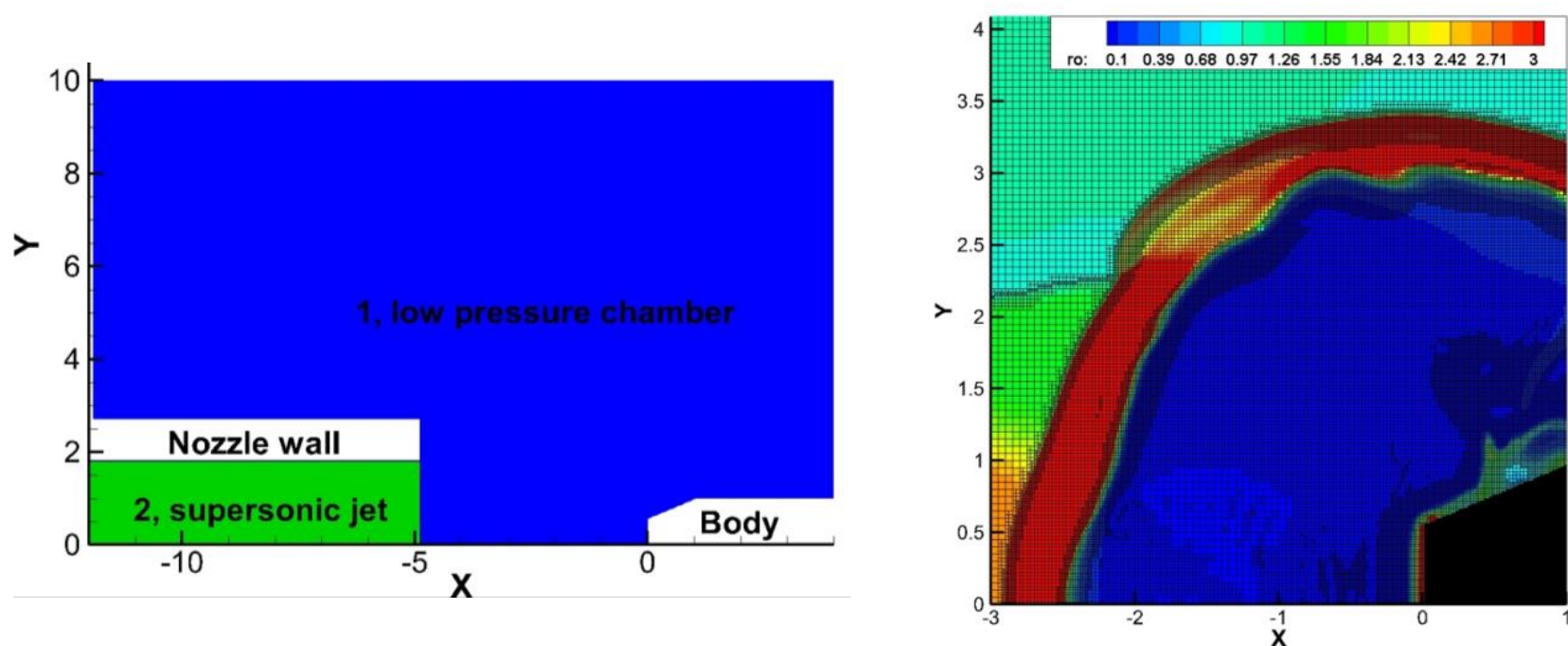

Fig. 2. Calculation area and local adaptive grid structure at an unspecified time.

Energy input is simulated using $q$ component in the energy conservation equation:

$$
\begin{gathered}
\frac{\partial U}{\partial t}+\frac{\partial F}{\partial x}+\frac{\partial G}{\partial y}=H+H_{q}, U=(\rho, \rho u, \rho v, e)^{T}, p=(\gamma-1) \rho e \\
F=\left(\rho u, \rho u^{2}+p, \rho u v,(e+p) u\right)^{T}, G=\left(\rho v, \rho u v, \rho v^{2}+p,(e+p) v\right)^{T} \\
H=\frac{\omega}{y}\left(-\rho v,-\rho u v,-\rho v^{2},-(e+p) v\right)^{T}, H_{q}=(0,0,0, q)^{T}
\end{gathered}
$$

In this paper, the energy discharge from [4] is represented by 3 energy impulses with constant power $q$ (every impulse has different $q$ ). The energy input area is a sphere with a radius $\bar{r}=1$ and center at $\overline{x_{0}}=-1.5, \overline{y_{0}}=0$. Normalized $\bar{q}=820$, derived from $q=$ $0.05 Q(5 \%), E=I_{\max } U, Q=E / V_{\text {sphere }}$. From Fig. 1, we assumed the following periodicity pattern:

1) 1st impulse $t \in[0 ; 35 m k s] \quad q=\bar{q}$

2) 2nd impulse $t \in[70 \mathrm{mks} ; 105 \mathrm{mks}] \quad q=\bar{q} / 2$

3) 3rd impulse $t \in[140 \mathrm{mks} ; 175 \mathrm{mks}] \quad q=\bar{q} / 8$ 


\section{Computation results on Cartesian grids with local adaptation}

Animations 1-4 show the flow structure evolution during and after energy input. All results are illustrated using Tecplot instruments [17]. Animation 1 shows pressure field distribution with streamtraces. Animation 2 presents the density field distribution. As can be seen from shadowgraphs calculated with Tecplot and presented on Animation 3, the process is unsteady and can be characterised by the interaction of a large number of discontinuities. Using such a visualization tool as a shadowgraph (Laplacian of density, $\nabla^{2} \rho$ ) makes it possible to effectively compare numerical results with physical experiment. Adaptive grid refinement and coarsement algorithm can be seen capturing them from Animation 4 which shows grid structure evolution. Mesh adaptation to the solution is conducted based on density field analysis. The presented animations allow conducting a comprehensive analysis of the flow structure evolution, correlating with changes in the computational grid.
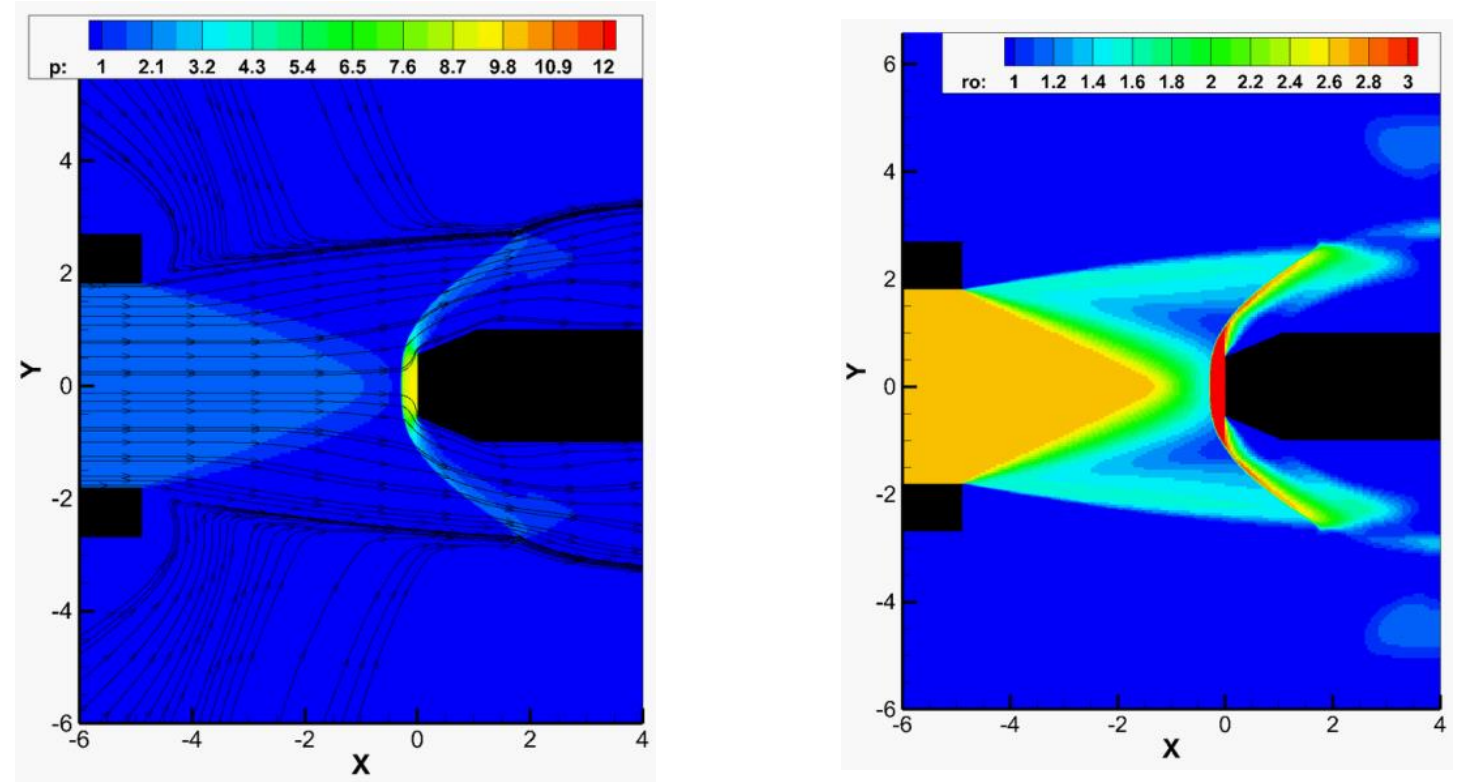

Animation 1 - pressure distribution and streamtraces (left), Animation 2 - density distribution (right)
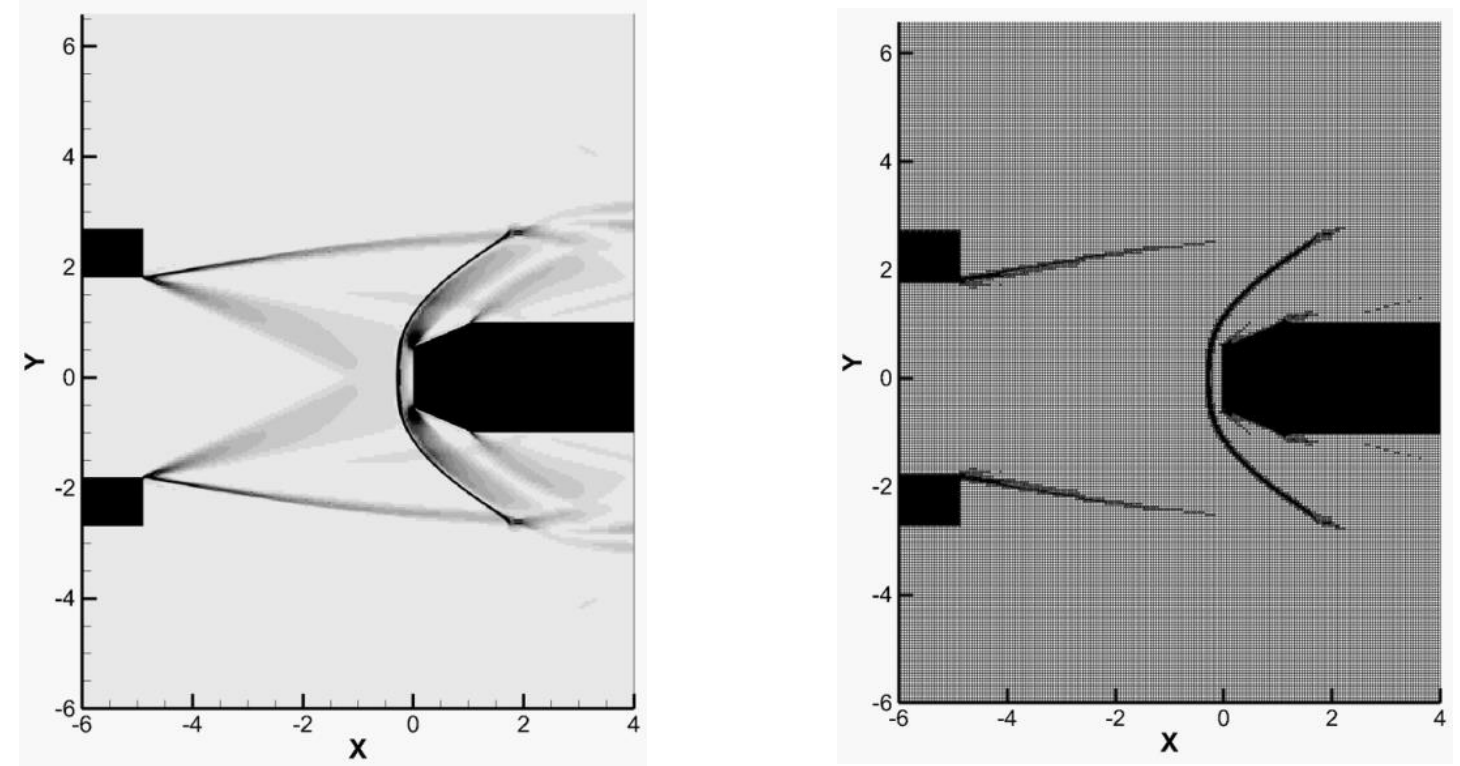

Animation 3 - shadowgraph (left), Animation 4 - grid structure (right) 

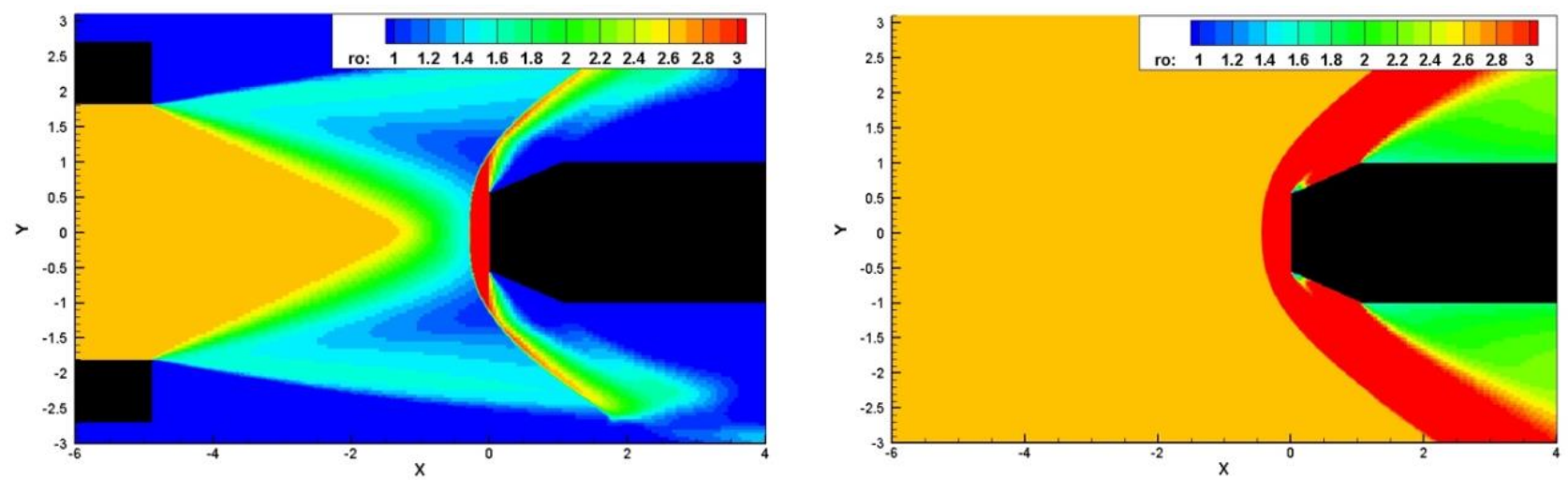

Fig. 3. Density distribution during underexpanded jet flow (left) and regular supersonic flow (right) at $\mathrm{t}=\mathrm{o}$.

Fig. 3 shows the structure of the flow before energy input. Jet boundary, oblique shock, expansion waves (characteristic of an underexpanded jet flow [13]) and bow shock wave can all be seen taking place before the body. The bow shock wave standoff distance is less than in the case of a regular supersonic flow with the same Mach number (Fig. 3). Due to presence of a body at this particular distance from nozzle, there is a non- barrel-shaped flow structure and a Mach disk

At $\mathrm{t}=5 \mu \mathrm{s}$ after the $1^{\text {st }}$ energy impulse starts a spherical shock wave and the contact discontinuity takes the form of an energy input area moving away from the center (Fig. 4). A rarefaction wave is formed from the discontinuity boundary and moves toward the center of energy input area. The energy source of the shock front closer to the body interacts with the bow shock wave increasing the body drag (Fig. 5).

At $t=12 \mu \mathrm{s}$, due to the fact that shock front of the jet energy source closer to it stays in place, while others continue to radiate from energy source (Fig. 6). The bow shock wave moves away from the body through the heated gas area. A structure reminiscent of the $2^{\text {nd }}$ type of shockimpact interaction according to Edney's classification [14] can be seen inside the shock wave energy source. A triple configuration takes place on the lateral part of the body.
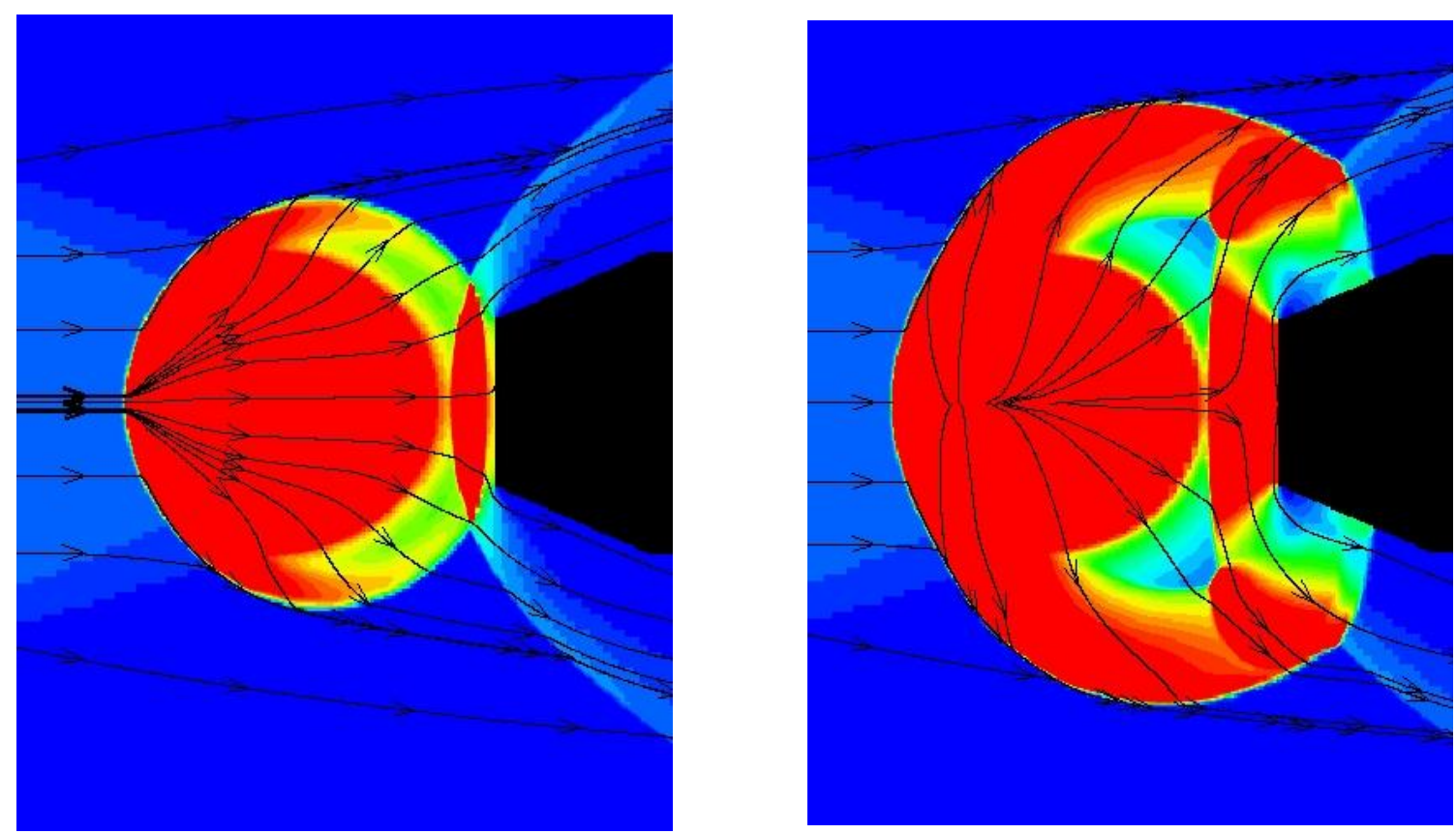

Fig. 4. Pressure distribution with stream traces at $t=5 \mu$ s (left) and $t=12 \mu$ s (right) after the beginning of the1st energy impulse. 


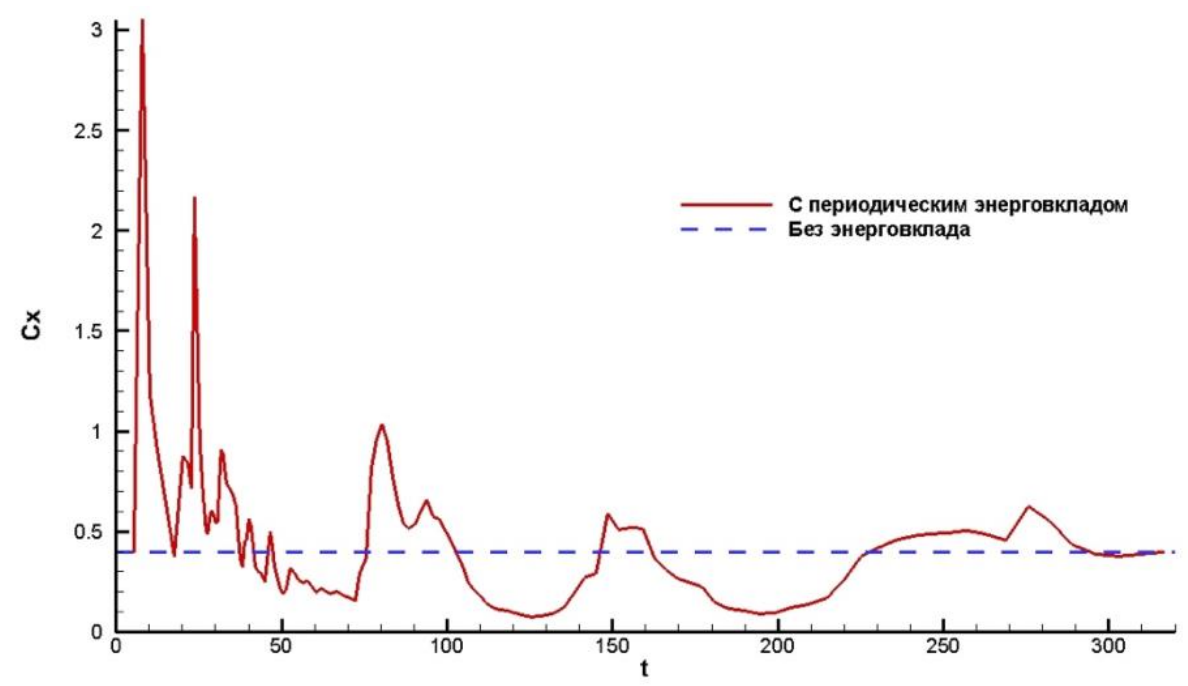

Fig. 5. Body drag coefficient during and after energy input.

After the $1^{\text {st }}$ energy impulse of the final energy source, the shock wave front continues to expand into the low-pressure chamber, but is weakened. After it reaches the boundary of the jet accelerates leads to the front curves and begins to interact with its own facing front the jet and the boundary of the jet (Fig. 6). The contact discontinuity of the energy source becomes unstable due to passage of the jet boundary and also starts to curve. The bow shock wave continued to move away from the body which leads to decrease in body drag below the level without energy input (Fig. 5). The shock front of the energy source facing the jet is pushed by the jet in the direction of the body due to the $1^{\text {st }}$ impulse ending. Small vortexes occur in front of the body, which is a characteristic of the interaction of the input energy with the blunt body [7] but they don't stay there and are pushed off the head part due to the periodic nature of energy input in this work. Fig. 7 shows a satisfactory comparison of numerical simulation and experiment on the placement of the shock wave and contact discontinuity placement [4].
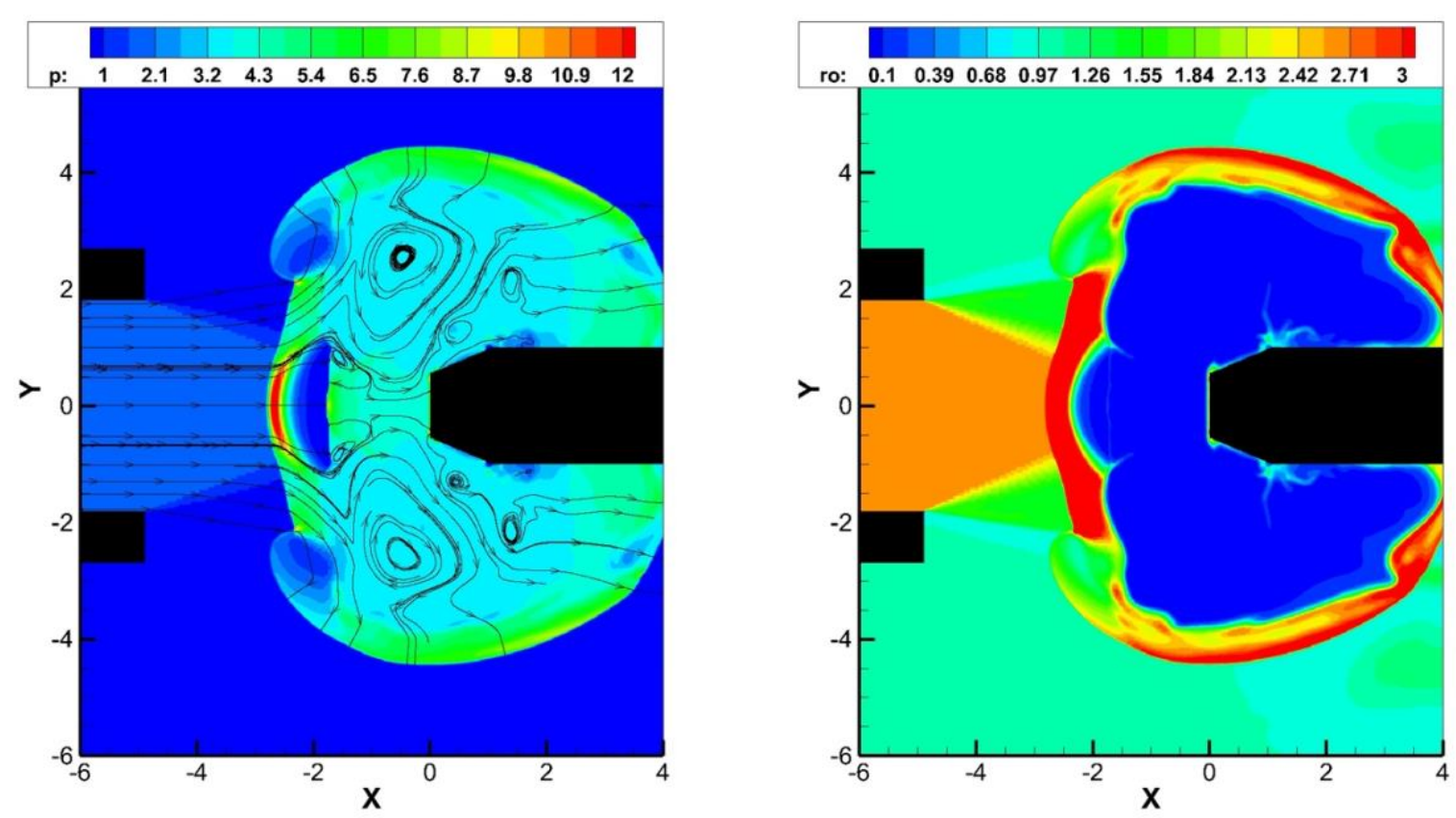

Fig. 6. Pressure distribution with stream traces (left) and density distribution (right) at $t=40$ $\mu$ s (the ${ }^{\text {st }}$ energy impulse ended). 

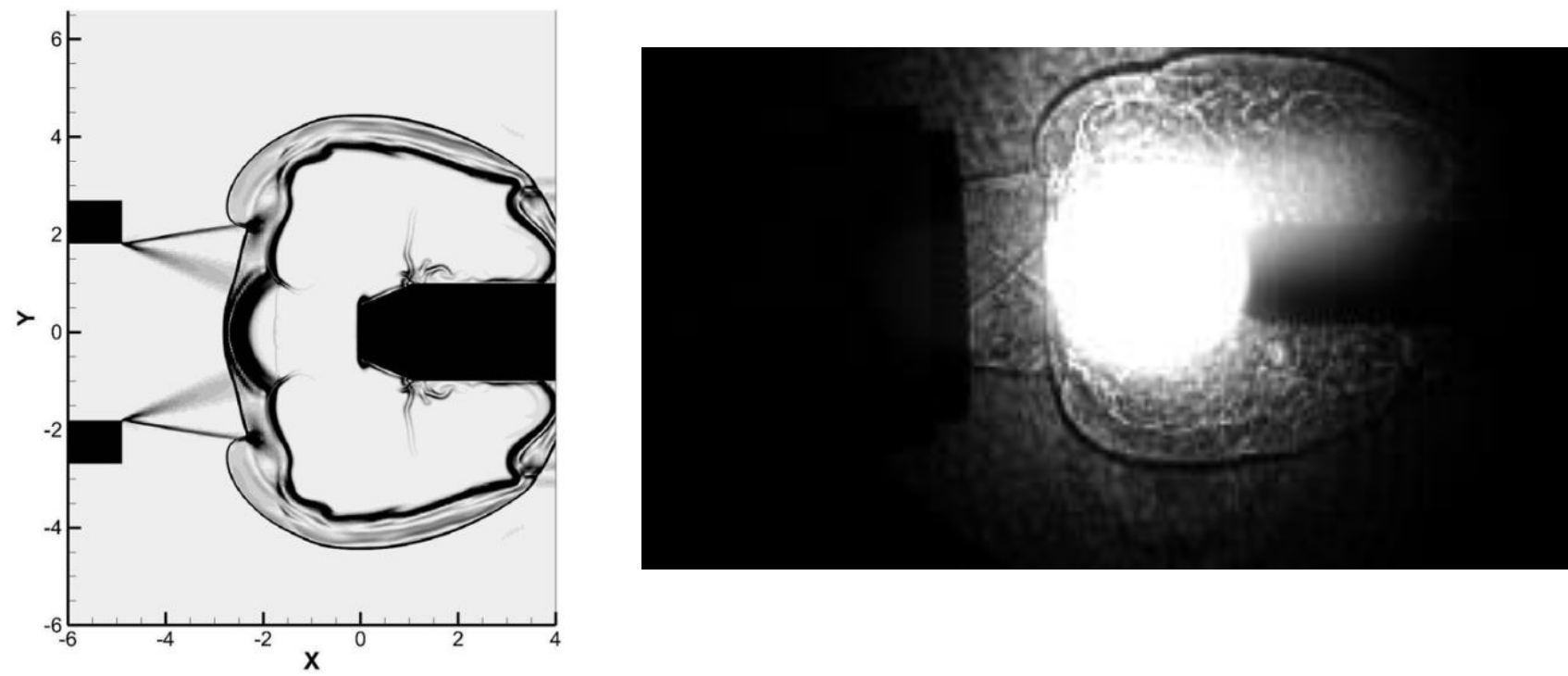

Fig. 7. Shadowgraph comparison between numerical simulation (left) and experiment [4] (right) at $\mathrm{t}=40 \mu \mathrm{s}$.

The $1^{\text {st }}$ shock energy impulse continues to interact with itself and the jet boundaries as a result of the $2^{\text {nd }}$ type the Edney configuration. The front of the $2^{\text {nd }}$ energy impulse shock reaches that configuration and starts to interact with it (Fig. 8). The front of the shock facing the body reaches the body and is reflected from its raising body drag (Fig. 5).
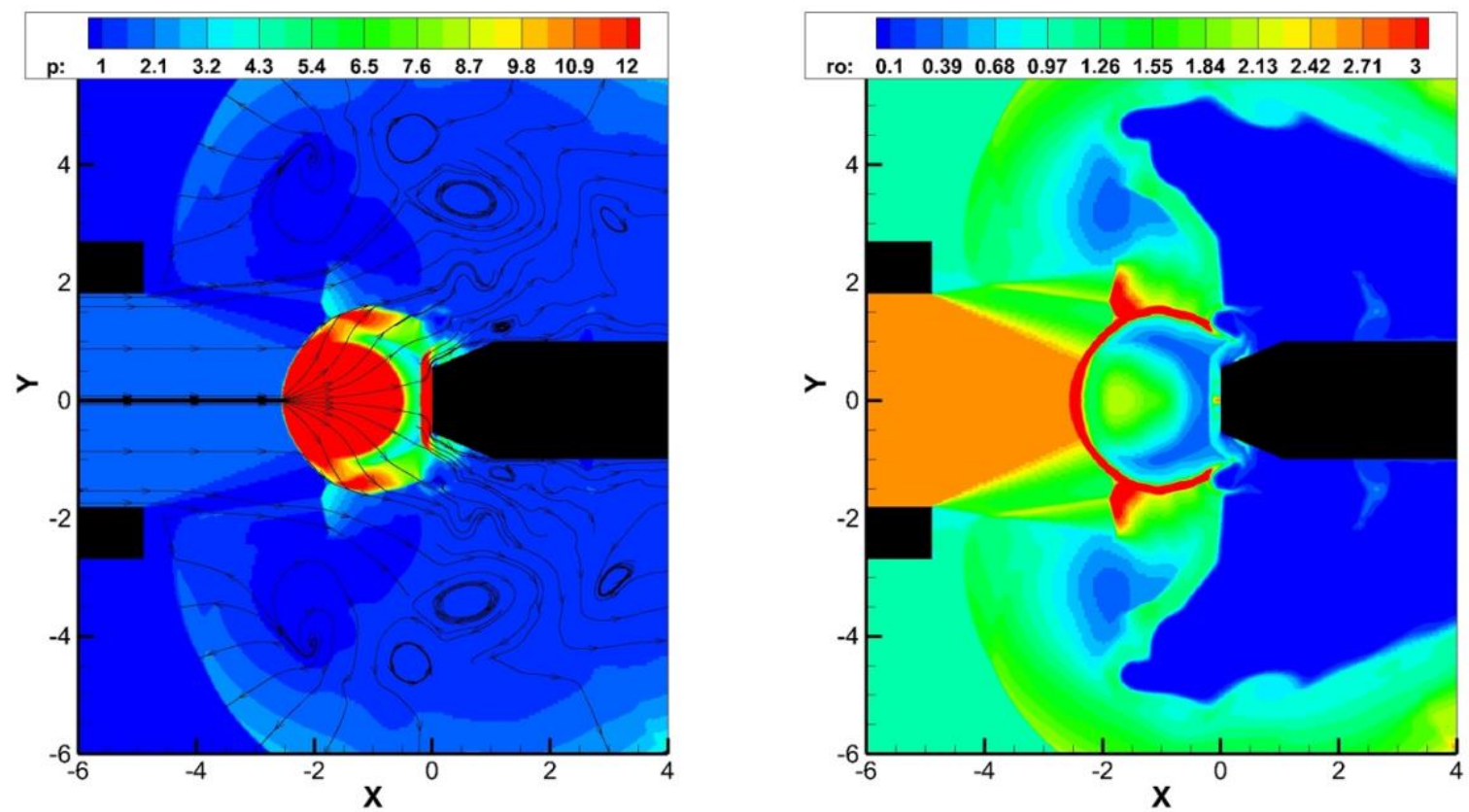

Fig. 8. Pressure distribution with stream traces (left) and density distribution (right) at $\mathrm{t}=8 \mathrm{o} \mu \mathrm{s}\left(10 \mu \mathrm{s}\right.$ after the beginning of the $2^{\text {nd }}$ energy impulse $)$.

At $t=107 \mu \mathrm{s}$, the $1^{\text {st }}$ energy impulse of the shock front reached the nozzle (Fig. 9). Reflected the 2nd impulse of energy shock as a bow shock wave, before that it moved away from the body, lowering the body drag below the level without energy input (Fig. 5). The shock wave of the $2^{\text {nd }}$ energy impulse starts to interact with the curved discontinuity of the $1^{\text {st }}$ contact of the impulse energy. The small vortex structures continue to occur near the body and get pushed off from it. 

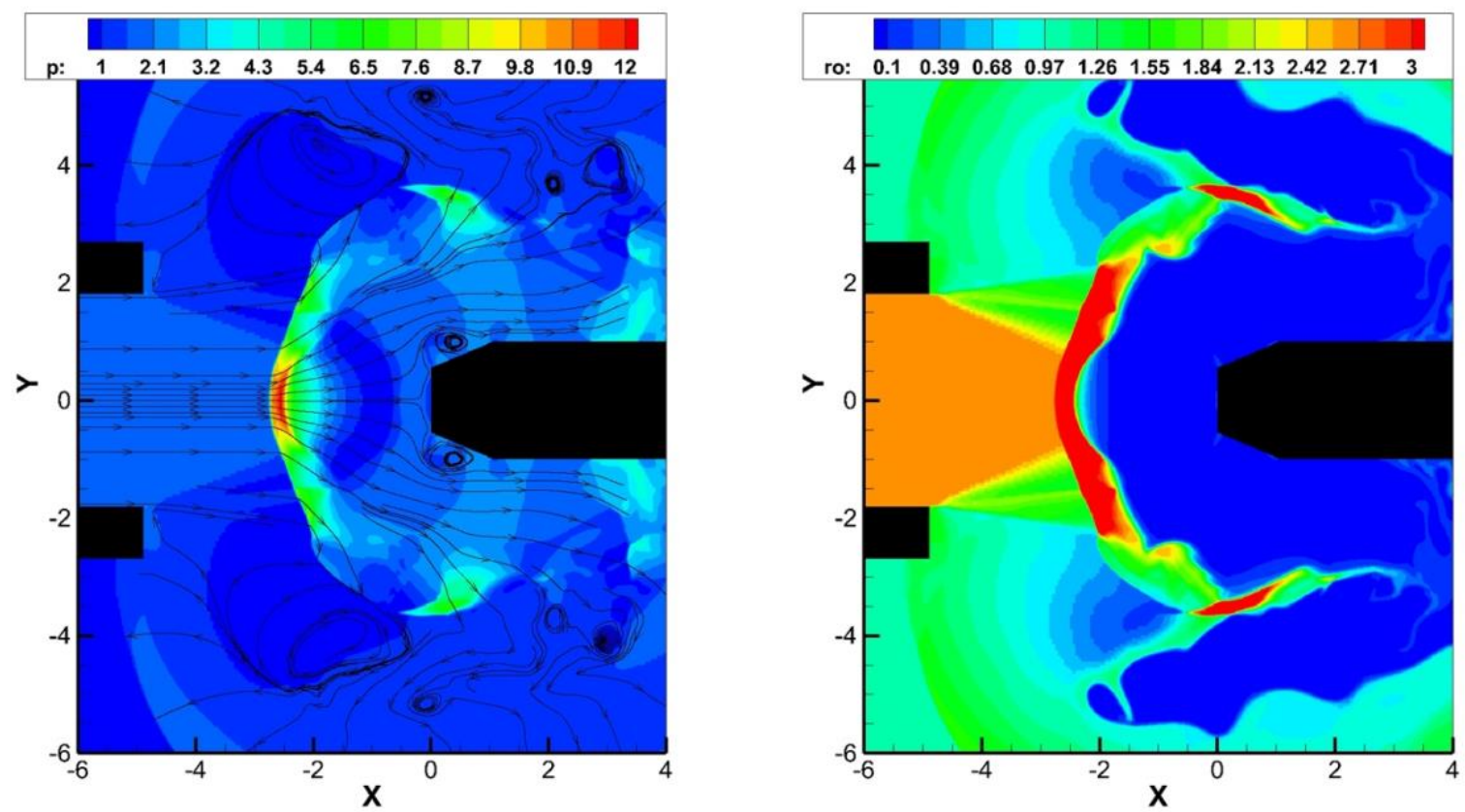

Fig. 9. Pressure distribution with stream traces (left) and density distribution (right) at $\mathrm{t}=$ $107 \mu \mathrm{s}$ (the $2^{\text {nd }}$ energy impulse ended).
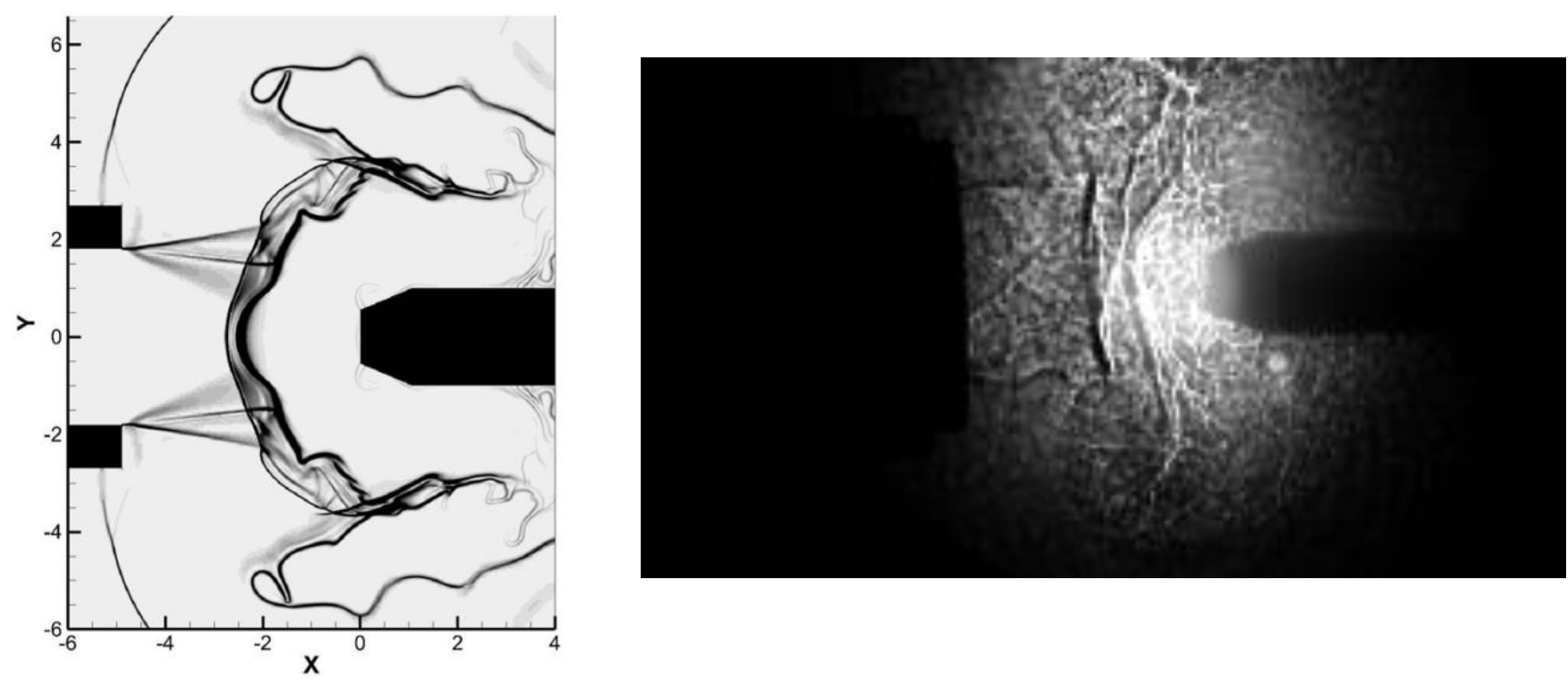

Fig. 10. Shadowgraph comparison between numerical simulation (left) and experiment [4]

(right) at $\mathrm{t}=107 \mu \mathrm{s}$. Figure 10.

Fig. 10 shows a comparison of numerical simulation and experiment [4] at $t=107 \mu$ s. The shock front of the 2nd energy impulse, facing the jet, and placement are similar, though some other structures aren't visible in the experiment which is probably due to inexact experimental conditions of numerical simulation.

The $3^{\text {rd }}$ energy impulse is the weakest of the three, which results in a less shock wave intensity (Fig. 11). The same trends as the $2^{\text {nd }}$ energy impulse can be seen here, such as interaction with previous impulse shock fronts, energy shock wave reflection from the body and subsequent increase in body drag. The shock wave interaction of the $2^{\text {nd }}$ energy impulse with the curved contact discontinuity of the $1^{\text {st }}$ energy impulse results in vortices and structures similar to the Richtmyer-Meshkov instability [15,16], which can occur at this type of interaction. 

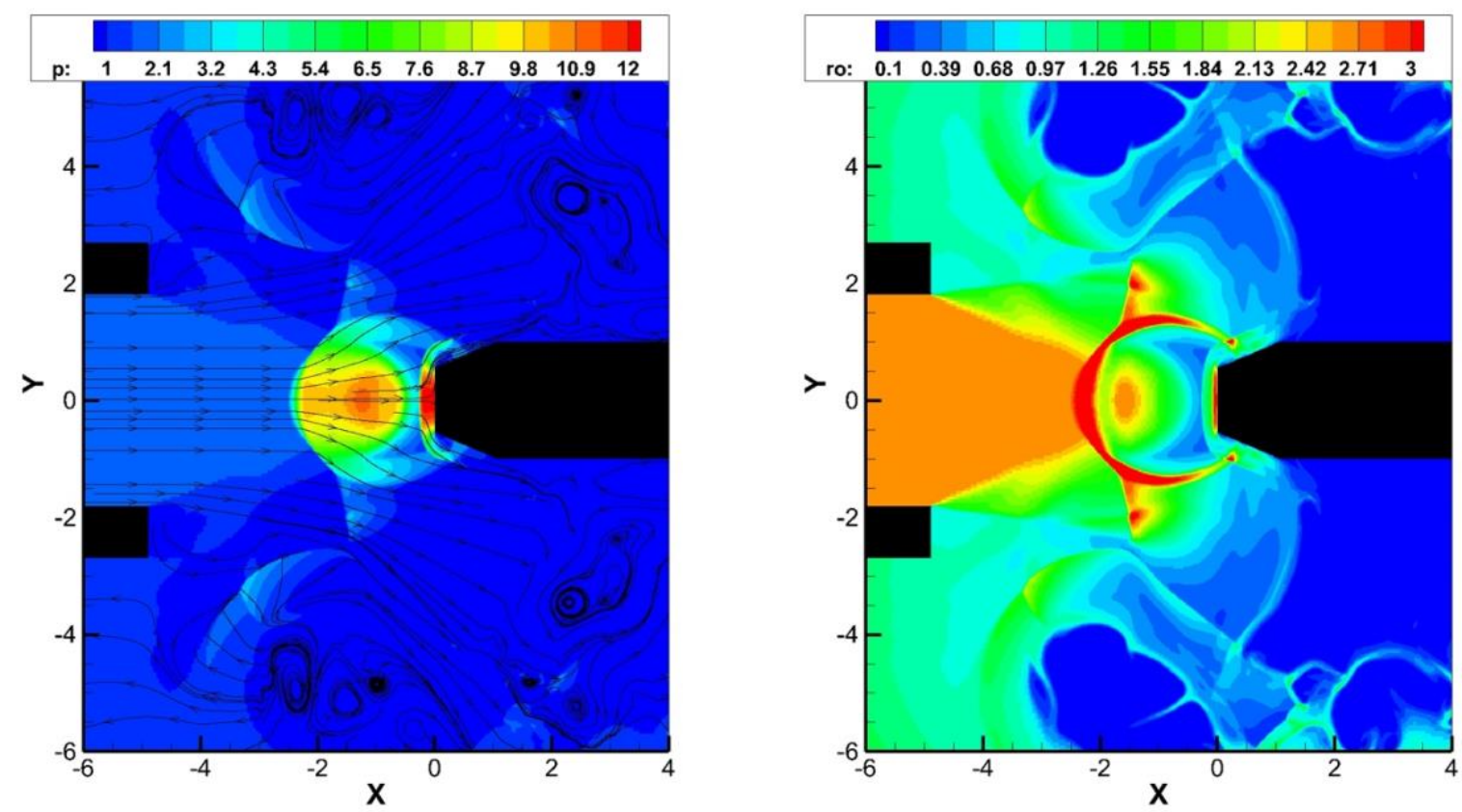

Fig. 11. Pressure distribution with stream traces (left) and density distribution (right) at $t=$ $152 \mu \mathrm{s}$ (12 $\mu \mathrm{s}$ after the start of the $3^{\text {rd }}$ energy impulse).

In the end, after all the energy impulses have ended, the flow starts to return to its pre-energy input state (Fig. 12). The bow shock wave takes form, the body drag establishes at a level without energy input. Structures from the interaction of various discontinuities move away from the body.
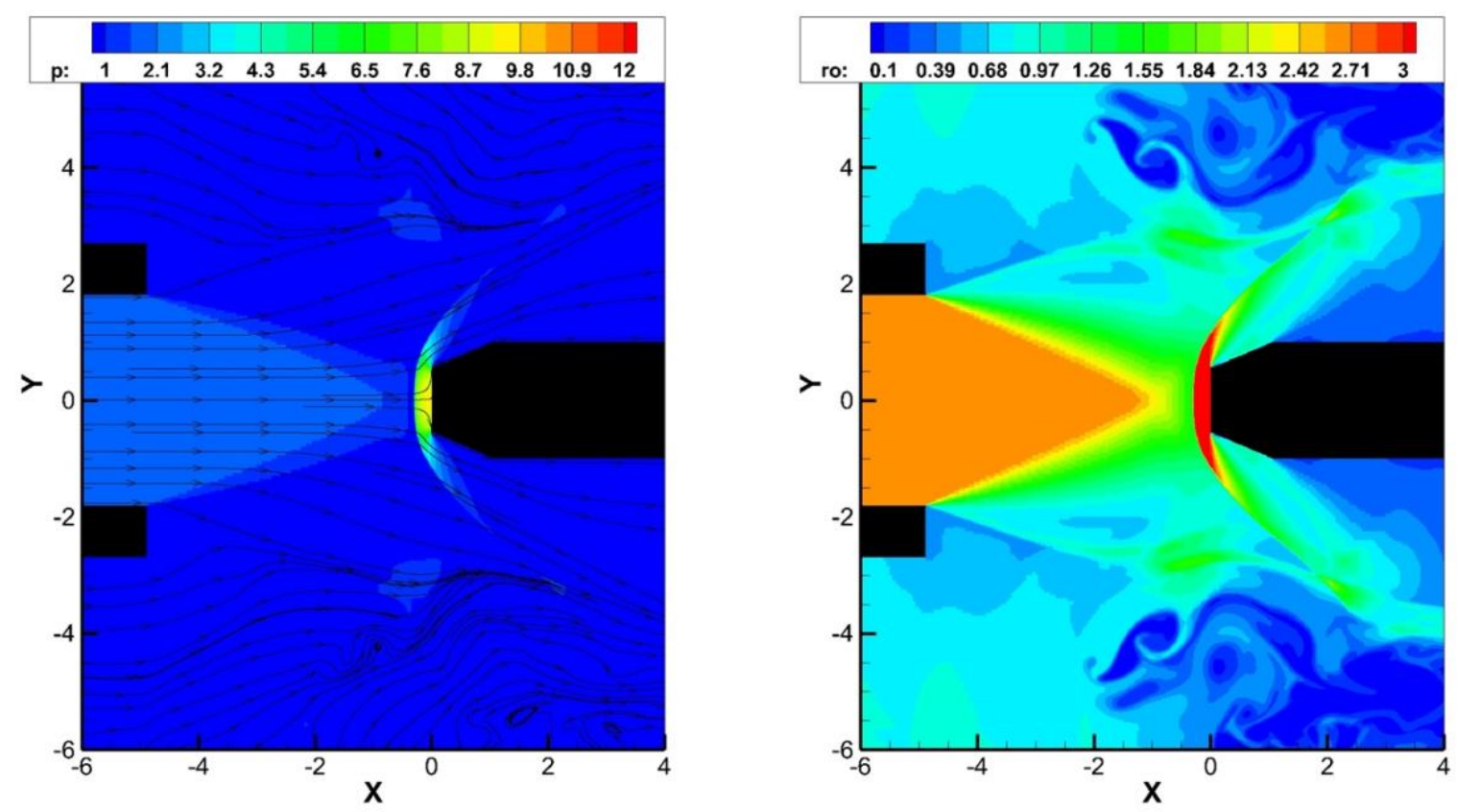

Fig. 12. Pressure distribution with stream traces (left) and density distribution (right) at $\mathrm{t}=$ $316 \mu \mathrm{s}$.

\section{Conclusion}

The flow structure evolution during the interaction of a supersonic underexpanded jet, blunt body and periodic energy input has been investigated using the free boundary method on local adaptive grids with adaptation based on wavelet analysis.The grid is restructured in accordance with various occurring discontinuities. Grid adaptation allows for significantly speed up the simulation. A regular uniform grid with the smallest size cells of the adaptive 
grid would have consisted of a more than 1 million cells, while the number of adaptive grid cells didn't exceed 350 thousand.

The periodic input of energy leads first to an increase in body drag (due to the energy source of the shock wave reaching the body), then to a decrease to a level lower than if no energy input was present. A large amount of discontinuities and their interaction with each other can be observed. Due to the flow around the body being non-uniform (the presence of under expanded jet and low-pressure chamber), shock waves occurring from the energy input become curved. Shock waves from previous energy inputs interact with those from the next ones and contact discontinuities, which results in structures similar to the RichtmyerMeshkov instability.

Visualization tools (instantaneous images and animations of gas-dynamic fields, stream traces and shadowgraphs) provide a deep understanding of the nature and dynamics of flow evolution and complex processes of shock interaction.

\section{References}

1. S. Leonov, V. Bityurin, A. Yuriev, S. Pirogov, B. Zhukov. Problems in energetic method of drag reduction and flow/flight control. 41st Aerospace Science Meeting and Exhibit, Reno, Nevada, USA, 6-9 Jan. 2003. AIAA Paper, 2003-35, 8p.

2. P.K. Tretyakov, A.F. Garanin, G.N. Grachev, V.L. Krainev, A.G. Ponomarenko, V.N. Tishchenko, and V.I. Yakovlev. Control of Supersonic Flow around Bodies by Means of High-Power Recurrent Optical Breakdown. Physics-Doklady, 1996, Vol. 41, No. 11, p. 566.

3. Yu.F. Kolesnichenko, V.G. Brovkin, O.A. Azarova, V.G. Grudnitsky, V.A. Laskov, I.Ch. Mashek. MW energy deposition for aerodynamic application. 41st Aerospace Science Meeting and Exhibit, Reno, Nevada, USA, 6-9 Jan. 2003. AIAA Paper, 2003-361, 11p.

4. I.A. Znamenskaya, D.S. Naumov, N.N. Sysoev, et al. Analysis of Dynamic Processes Occurring during Generation of Plasmoid Formations in a Supersonic Flow. Tech. Phys., 2019, vol. 64, no.6, pp. 802-806.

5. P. Yu. Georgievskii, V. A. Levin. Control of the flow past bodies using localized energy addition to the supersonic oncoming flow. Fluid Dyn., 2003, vol. 38, pp. 794-805.

6. V. P. Gordeev, A. V. Krasil'nikov, V. I. Lagutin, and V. N. Otmennikov. Experimental study of the possibility of reducing supersonic drag by employing plasma technology. Fluid Dyn., 1996, vol. 31, pp. 313-317.

7. I.Y. Kudryashov, A.E. Lutsky, Y.V. Khankhasaeva. Numerical simulation of the influence of energy deposition on the base flow. Mathematical Models and Computer Simulations, 2016, vol. 8, no. 2, pp. 207-218.

8. A. L. Afendikov, A. E. Lutsky, I. S. Menshov, V. S. Nikitin, Ya. V. Khankhasaeva. Chislennoe modelirovanie vozvratnogo techeniya pri razdelenii dvizhushikhsya so sverkhzvukovymi skorostyami tel [Numerical simulation of recirculation flow during supersonic separation of moving bodies]. Matem. Mod., 2019, vol. 31, No. 9, pp. 21-38. [In Russian]

9. Ya.V. Khankhasaeva, A.L. Afendikov, A.E. Lutsky, I.S. Menshov, V.S. Nikitin. Simulation and visualization of the flow around blunted body in the wake of supersonic ejecting pellet. Scientific Visualization, 2019, vol. 11, no. 1, pp. 57 - 69. (URL: http://svjournal.org/2019-1/o5/\#_Toc2564272)

10. A.L. Afendikov, A.A. Davydov, I.S. Menshov, K.D. Merkulov, A.V. Plenkin, Ya.V. Khankhasaeva. Adaptivnye veivletnye algoritmy dlya resheniya zadach gidro- i gazovoi dinamiki na dekartovykh setkakh [Adaptive wavelet algorithms for solving problems of hydro- and gas dynamics on Cartesian grids]. M.: IPM im. M.V. Keldysha, 2016. - $232 \mathrm{~s}$. ISBN 978-5-98354-030-9. [In Russian]

11. I.S. Menshov, M.A. Kornev. Free-boundary method for the numerical solution of gasdynamic equations in domains with varying geometry. Mathematical Models and Computer Simulations, 2014, vol. 6, no. 6, pp. 612-621. 
12. I. S. Menshov, P. V. Pavlukhin, Efficient Parallel Shock-Capturing Method for Aerodynamics Simulations on Body-Unfitted Cartesian Grids. Computational Mathematics and Mathematical Physics, 2016, vol. 56, no. 9, pp. 1651-1664.

13. V.M. Boyko, A.V. Dostovalov, V.I. Zapryagaev, I.N. Kavun, N.P. Kiselev, A. A. Pivovarov. Investigation of supersonic nonisobaric jet structure. TsAGI Science Journal, 2010, vol. 41, no. 2, pp. 187-205.

14. B. Edney. Anomalous heat transfer and pressure distributions on blunt bodies at hypersonic speeds in the presence of an impinging shock. Aeronautical Research Institute of Sweden, Report 115, Stockholm (1968). (URL: https://www.osti.gov/biblio/4480948)

15. E.E. Meshkov. Instability of the interface of two gases accelerated by a shock wave. Fluid Dynamics, 1969, vol. 4, no. 5, pp. 101-104.

16. R.D. Richtmyer. Taylor instability in shock acceleration of compressible fluids. Comm. Pure. Appl.Math., 1960, vol.13, pp. 297-319.

17. Computational fluid dynamics and numerical simulation software package Tecplot, http://www.tecplot.com/ 